\title{
A Interface e as suas dimensões na perceção de credibilidade e confiança na e-Health. Proposta de uma estrutura para análise da Interface
}

\author{
The Interface and its dimensions in e-Health credibility and \\ trust perception Interface evaluation structure proposal
}

\author{
Andreia Pinto de Sousa, Ana Margarida Almeida
}

Interface; Confiança; Interação humanocomputador; e-Health; Credibilidade

Interface; Trust; Humancomputer interaction; e-Health; Credibility

\begin{abstract}
A Interface é incontornável quando se estudam os sistemas de informação. Com este artigo, pretendemos contribuir para o aumento do conhecimento sobre os processos de apresentação de informação de saúde online e, sobre as relações do utilizador com a Interface. A proposta aqui apresentada consiste na divisão da interface em cinco dimensões (visual, arquitetura da informação, interação, presença social e experiência de utilização) para um maior conhecimento da relevância de cada uma das dimensões e dos seus elementos no estudo da credibilidade e confiança online. Apresenta-se também um modelo de análise que serve como base de construção dos instrumentos de análise para a recolha de dados que caraterizam os utilizadores, e os seus comportamentos relativamente ao acesso e participação e avaliam a sua perceção de credibilidade e de confiança em websites partindo da análise de cada uma das dimensões da Interface.
\end{abstract}

The Interface is essential when studying information systems. With this article, we aim to contribute to the increase of knowledge about online health information processes and, on the user's relationship with the Interface. The proposal presented here divides the interface into five dimensions (visual. information architecture, social presence and user experience) for a better understanding of the relevance of each dimension and its elements in the study of online credibility and trust. Its also presented an analysis model that serves as a framework for analytical instruments to collect data that characterizes users and their behavior, regarding access and participation and also their perception of credibility and trust in websites starting from the analysis of each Interface dimension.

\section{Introdução}

Num contexto em que a Web é o lugar para onde confluem os indivíduos e os serviços é importante que se investiguem, neste novo contexto online, relações que têm sido estudadas e caraterizadas em contextos offline como é o caso da credibilidade e da confiança. Conscientes de que a área da e-Health é uma das que mais tem 
crescido nos últimos tempos, quer seja em volume de oferta, como de procura, e que esse mesmo enquadramento se carateriza pela criação de um novo paradigma de relação entre os seus intervenientes (utente e profissional de saúde), interessa-nos compreender e avaliar como se criam relações de credibilidade e confiança online, especificamente na área da saúde. No que toca à construção da relação de confiança, a Interface assume sempre o papel de depositário representado, na maior partes das vezes, uma entidade ou serviço. Com o objetivo final de enunciar princípios de design para a credibilidade e a confiança na e-Health, propomos uma sistematização da Interface em cinco dimensões partindo das quais desenvolvemos um modelo de análise e com base na metodologia de desenho centrado no utilizador desenvolvemos instrumentos de análise - um inquérito por questionário e um "web browser plugin" e sessões com eye tracker. Com base nestes instrumentos pretendemos não só caraterizar os utilizadores e os seus comportamentos no que diz respeito ao acesso, uso, comunicação e partilha de informação de saúde como, também, avaliar sua perceção de credibilidade e confiança em websites partindo da análise da relevância de cada uma das dimensões da Interface.

\section{Da interface à credibilidade e confiança na e-Health}

A Interface é um elemento mediador nas relações entre humanos ou nas relações interpessoais e nas relações entre humanos e as máquinas. Consideramos que a Interface representa um elemento determinante no tipo e na qualidade das interações que esta envolve. A Interface não se limita ao modo de apresentação de um objeto e de um espaço. Em ultima estância, define também as interações que acontecem entre as pessoas. Nas palavras de Sá (2010), “a Interface é um espaço híbrido que sintetiza heterogeneidades provenientes das entidades colocadas em relação. A sua materialidade plástica é constituída pela hibridação entre os sistemas a relacionar, sendo limitada por eles e limitando-os, efetivando-se e ganhando forma pela ação" (Sá, 2010, pp. 209-210). Partindo desta perspetiva ampla da Interface em que esta determina a forma como experienciamos e definimos o mundo em que vivemos, neste artigo iremos focar-nos essencialmente na Interface entre $o$ utilizador e as plataformas digitais, muitas das vezes denominada por Interface Gráfica [Graphic User Interface], designação que não será adotada neste estudo porque, no nosso entender, a utilização da expressão gráfica parece limitar demasiado a Interface questões gráficas ou visuais. Para (Raskin, 2000, p. 2)a Interface é a forma como concretizamos tarefas com um produto e como ele responde às nossas ações.

O ponto de contato entre a informação disponível na Web e os indivíduos continua a ser a interface, sendo nesta e através desta que se estabelece o ponto de partida para a perceção e criação dos processos 
de credibilidade e de confiança. Considerando a Interface como responsável máxima da experiência através da qual interagimos com os outros, os objetos e os espaços, assumindo o papel de mediadora da comunicação e influenciando assim a relação entre os sistemas digitais e as pessoas, torna-se necessário conhecer um pouco mais sobre essa influência. Em 2003, Fogg, apresentou o resultado do estudo intitulado "How do users evaluate the Credibility of Web sites?" (Fogg, 2003) no qual, ao contrário do que era habitual, a análise da credibilidade em websites não se focou apenas na informação. Partindo de um grupo predefinido de websites, apresentado aos participantes do estudo, foi solicitado que estes atribuíssem a cada um deles um rating em termos de confiança. Este rating foi acompanhado de comentários sobre a credibilidade de cada um dos sites. A análise dos comentários fez-se recorrendo à teoria da Proeminência - relativo à perceção de algo, e da Interpretação - relativa ao julgamento, para a perceção de credibilidade. Os resultados deste estudo são reveladores relativamente à influência da Interface na perceção da credibilidade tornando-se um trabalho de referência incontornável no estudo da credibilidade online. Igualmente importante para enquadrar esta problemática é o estudo de Wang \& Emurian (2005) que propõem uma organização de caraterísticas de design que contribuem para a confiança online. Estes, identificaram quatro dimensões indutoras de confiança através da interface: o design visual, o design de pistas sociais, o design da arquitetura de informação e o design de interação. Também Robins, Holmes, e Stansbury (2009) isolaram a análise da credibilidade da informação da análise da credibilidade através design visual. Partindo de estudos anteriores (Fogg et al., 2003; Robins, Holmes, \& Stansbury, 2009) demonstram que o design visual tem impacto na perceção de credibilidade. Os autores concluíram que existe uma relação entre o design visual de um website de consumo de informação sobre a saúde e a perceção de credibilidade da informação que este contém, mas não conseguiram demonstrar as razões para essa relação.

Com efeito, e apesar ter sido alvo da atenção de diferentes estudos, a problemática do estudo da confiança online ainda necessita de mais investigação, como comprova o levantamento de estudos relacionados com o design de websites e a confiança inicial, desenvolvido por Karimov, Brussel, Brengman e Hove (2011). Como outros aqui apresentados, este estudo foca-se essencialmente na área do e-Commerce: partindo de uma extensiva revisão de literatura (desde 1996 a 2010), o estudo reconhece que não existe um standard para a avaliação das dimensões de websites e propõe um modelo conceptual de classificação das dimensões de design que induzem a perceção de confiança: o design visual, o design de pistas sociais e o design de conteúdos. Com base nesta organização, os autores reuniram todos os resultados, encontrados na literatura, do impacto destas dimensões na criação de confiança inicial, tendo os resultados obtidos demonstrado que o impacto das dimensões de design na confiança inicial de websites está pouco investigado. 


\subsection{Credibilidade e confiança}

O conceito de confiança é complexo e abstrato tornando difícil a sua definição, e consequentemente, a identificação dos elementos que o envolvem. Pode definir-se como uma crença ou dependência numa qualidade ou atributo de uma pessoa ou objeto (Furman, 2009). Pesquisas em diferentes domínios indicam que a confiança constrói-se gradualmente através de diferentes interações e que permite às pessoas adquirirem crenças sobre a outra pessoa ou objeto - tendo a confiança como base a interação, pressupõe que existem sempre duas partes envolvidas no processo. A confiança deve, pois, ser entendida como uma crença individual de que as outras pessoas se vão comportar de uma forma ética e socialmente adequada e ainda como uma expetativa de que aqueles em que se escolhe confiar não se vão aproveitar da situação (Chow \& Angie, 2006; Gefen \& Karahanna, 2003). Uma vez caraterizada a confiança será necessário perceber a sua relação com a credibilidade. Assumindo que na literatura académica e profissional os termos credibilidade e confiança são utilizados imprecisamente, e que, apesar de serem termos relacionados não são sinónimos, Fogg (2003) debruçou-se sobre o tema da credibilidade definindo-a como uma qualidade percebida, não residindo num objeto, numa pessoa ou num pedaço de informação, em comparação com a confiança, que se define como uma crença positiva sobre a confiabilidade numa pessoa, objeto ou processo. Este autor compara a credibilidade como a beleza, pois está nos olhos do espetador; não consegue ser tocada, vista ou até ouvida; existe apenas quando se faz uma avaliação de uma pessoa, objeto ou de um pedaço de informação e é composta por duas dimensões chave. Estas duas dimensões definidas pelo autor, e confirmadas por outros investigadores, são a confiança e a perícia que, embora sejam ambas dimensões da credibilidade, não estão diretamente relacionadas entre si, podendo existir perícia sem confiança ou vice-versa (Fogg, 2003, p. 122).

\subsection{A confiança e a credibilidade na Web}

À exceção de alguns estudos conduzidos por Fogg, no Stanford Research Lab, que utilizaram websites de saúde para analisar o seu impacto na credibilidade superficial, os estudos sobre a confiança e a credibilidade na Web têm-se focado essencialmente na área do e-Commerce, razão pela qual enquadramos necessariamente esta secção nos resultados obtidos com investigações conduzidas no campo do e-Commerce.

Wang e Emurian (2005) focaram-se no estudo do conceito de confiança em diferentes disciplinas (como a Psicologia, Filosofia, Gestão e Marketing) com a finalidade de a caraterizar offline e online. Na confiança denominada como "confiança offline", encontraram quatro caraterísticas frequentemente observadas e aceites por investigadores que são: o fiduciário e o depositário, a 
vulnerabilidade, a ação produzida e a questão subjetiva. A confiança offline partilha de caraterísticas similares com a confiança online, havendo, no entanto, distinções importantes que são únicas deste ambiente. No que respeita ao fiduciário e depositário, as duas partes continuam a ser essenciais num contexto online, sendo o papel de fiduciário maioritariamente ocupado pelo utilizador e o papel de depositário por um website que representa uma entidade. Será importante relembrar que muitas vezes, a tecnologia (e em especial a Web) é ela própria um objeto de confiança, ainda que a complexidade e o anonimato, associados aos serviços Web (e.g. websites de e-Commerce), façam com que os fornecedores e ou vendedores possam comportar-se de forma imprevisível, aumentando assim o fator de vulnerabilidade, sendo a perda de dinheiro e a perda de privacidade as duas principais preocupações em torno da violação de confiança online. Do ponto de vista da ação produzida, e porque os níveis de confiança do utilizador nos fornecedores e ou vendedores online conduzem necessariamente a dois tipos de ação (por um lado, a compra de um produto fornecendo informação sobre o cartão de crédito e dados pessoais e, por outro, utilizar o website como vitrine), qualquer umas destas ações é positiva para o fornecedor ou comerciante (Wang \& Emurian, 2005). No caso da e-Health, partindo da informação sobre saúde disponível nos websites, as ações produzidas poderão estar relacionadas com a tomada de decisão e a prevenção de saúde. Um elemento que estará sempre presente numa relação de confiança é a questão subjetiva que, tal como na confiança offline, está diretamente relacionada com diferenças individuais e fatores de contexto. A confiança online é inerentemente uma matéria subjetiva; já o nível de confiança necessário para cada indivíduo fazer uma transação online é diferente (Wang \& Emurian, 2005). No caso da e-Health esta questão subjetiva aplica-se igualmente à questão da tomada de decisão e da prevenção.

Como demonstrado por Fogg (2003)., as pistas sobre a presença de pessoas reais na Web, assim como a relação da informação com as suas fontes para que as pessoas possam confirmar a qualidade da informação, são perceções de confiança que aumentam a credibilidade. No que toca às perceções de perícia, podem destacar-se as respostas obtidas no apoio ao cliente e o feedback obtido através de email, quando são feitas transações online, assim como referência de quem são os autores dos textos. Nesta dimensão da perícia, parece fundamental atender à importância do aspeto visual dos websites e aplicações que, juntamente com aspetos de dimensão técnica e de experiência de utilização criam uma espécie de efeito de halo, contribuindo, de forma convergente e articulada, para uma perceção de maior credibilidade. A credibilidade e a confiança online estão, pois, diretamente relacionadas com a estrutura do meio de comunicação utilizado que no caso dos meios digitais, se materializa na interface. 
Deste modo, a informação afeta a estrutura que a contém e a estrutura afeta a própria informação, tal como o princípio da recursão organizacional preconiza (Morin, 2011). A mediação tecnológica entre os produtos e serviços influencia diretamente a perceção de confiança do individuo. Nos processos de análise da credibilidade e da confiança online importa, pois, observar com especial atenção a componente da vulnerabilidade que pode ter uma maior magnitude devido ao eventual anonimato de um dos atores na relação, podendo tal provocar a perda de confiança e de credibilidade. É neste contexto que nos parece pertinente investigar estratégias de design que possam contribuir para o aumento da credibilidade e da confiança online.

\subsubsection{Modelos para o estudo da confiança em sistemas de informação}

A proliferação de websites institucionais e de vendas na Web permitiu e impulsionou o aparecimento de muitos estudos sobre os processos de perceção e de avaliação de usabilidade, de satisfação e de confiança, entre outros. Como já referimos anteriormente, muitos destes estudos têm sido elaborados no contexto do e-Commerce e focam um dos problemas mais difíceis de analisar: a confiança e a sua relação com outras variáveis que influenciam diretamente a perceção do indivíduo (Riemenschneider et al., 2009). Nesse sentido têm sido recorrentes os estudos que pretendem aprofundar conhecimento nesta área; um dos modelos mais referenciado é o Information Systems Sucess de DeLone \& McLean, (1992) que apresenta uma abordagem multidimensional à avaliação de um sistema de informação e estrutura-o em seis categorias principais, apresentando uma relação entre cada uma delas. A Qualidade do Sistema e a Qualidade da Informação, em conjunto ou individualmente, afetam o Uso e a Satisfação do Utilizador; consequentemente, o Uso afeta positivamente ou negativamente o grau de Satisfação do Utilizador e a quantidade de Uso. A relevância deste estudo relaciona-se com a sua importância para estudos posteriores, não abordando especificamente a questão da confiança mas explorando questões em torno do design e, consequentemente, da interface, como a Qualidade do Sistema, o Uso e a Satisfação do Utilizador. Um dos estudos decorrente deste modelo é o proposto por Riemenschneider et al., (2009) que analisa a Perceção do Impacto Individual da Web com a Confiança, atuando como moderador entre a Usabilidade e a Perceção do Impacto Individual e entre a Satisfação e a Perceção do Impacto Individual. A Perceção do Impacto Individual é o efeito suposto da informação no comportamento do utilizador (DeLone \& McLean, 1992). Este estudo concluiu que, quando se consideram efeitos diretos, a Usabilidade/Satisfação têm influência na Perceção do Impacto Individual da Web e que existe uma forte relação entre Utilidade e Satisfação. Relativamente à Confiança, os resultados confirmam que esta influencia diretamente a Perceção do Impacto Individual da 
Web e a relação entre Usabilidade/Satisfação. Assim, quanto maior o nível de confiança que um indivíduo tem na Web, mais facilmente ele encontrará a combinação Usabilidade/Satisfação que, por sua vez, influenciarão a Perceção do Impacto Individual (Riemenschneider et al., 2009). Neste estudo conclui-se que a Usabilidade/Satisfação e a Confiança influenciam diretamente a Perceção do Impacto Individual da Web e que a Confiança modera a relação Usabilidade/Satisfação e a Perceção do Impacto Individual. Du \& Zhao, (2009) apresentam duas correntes de pesquisa adotadas como base para a fundamentação teórica da maioria das pesquisas sobre a Confiança em sistemas de informação: Theory of Reasoned Action (TRA) e Technology Acceptance Model (TAM). A TRA defende que os utilizadores têm geralmente duas crenças, num contexto Web sendo elas: (i) Crença de Uso - relacionada com a sua perceção do sistema; (ii) Crença de Confiança - que se refere à confiabilidade da organização em que o sistema reside. O TAM sugere dois determinantes para a intenção de uso: (i) Perceived Usefulness (PU) - medida em que os utilizadores percebem que a utilização de um sistema de informação irá melhorar o seu desempenho de trabalho; (ii) Perceived Ease of Use (PEOU) - medida em que a utilização de um sistema de informação requer muito esforço. Com base nestas duas correntes, Du e Zhao (2009) desenvolveram um modelo que tem como objetivo analisar os fatores que influenciam e contribuem para a confiança do utilizador final em sistemas de informação na Web. A análise dos dados recolhidos neste estudo demonstrou que a Facilidade de Uso percebida, a Utilidade percebida, a Familiaridade e a Normalidade contribuem para a Confiança do utilizador e que, por sua vez, a Confiança do utilizador final tem impacto direto na sua intenção de utilizar o sistema.

Em síntese, diferentes estudos já realizados testaram diferentes fatores que podem contribuir para a confiança em sistemas de informação, nomeadamente: a Usabilidade; a Facilidade de Uso; a Satisfação do Utilizador; a Qualidade da Informação; a Qualidade do Sistema; a Utilidade; a Interface Gráfica; o Desenho gráfico; o Desenho da estrutura; o Desenho dos conteúdos e o desenho de Desenho de pistas sociais. No contexto do presente estudo, entendemos todos estes fatores como elementos integrantes ou dependentes da Interface com o utilizador e da experiência de utilização (User Experience) e como dimensões chave na análise da qualidade dessa experiência. A interface é, do nosso ponto de vista, uma componente de materialização fundamental destes processos, no contexto das atuais dinâmicas de utilização de aplicações e serviços Web.

\section{3 e-Health}

A e-Health define-se como um campo emergente que se situa na interseção da informática médica com a saúde pública e dos negócios e refere-se à prestação de informação de serviços de saúde através da 
Internet e tecnologias relacionadas. Num sentido mais amplo este termo carateriza-se não só por um desenvolvimento técnico mas também por um estado de espírito, uma forma de pensamento e um compromisso com uma rede de pensamento global para melhorar os cuidados de saúde a nível local, regional e mundial, utilizando às tecnologias de informação e comunicação (Eysenbach, 2001). No contexto do atual cenário de reconfiguração dos cuidados de saúde para o século XXI, Fortney et al. (2011) propõem quatro novos tipos de prestação de cuidados de saúde, de mediação online, que devem ser considerados, para além dos já conhecidos encontros face-a-face entre o paciente e o prestador de cuidados de saúde: (i) Encontros síncronos entre o paciente e o prestador de cuidados de saúde; (ii) Comunicação assíncrona entre o paciente e o prestador de cuidados de saúde; (iii) Comunicação entre pares; (iv) Interações síncronas entre os pacientes e aplicações digitais de saúde. Todas estas tipologias são mediadas por plataformas digitais e gozam de algumas caraterísticas que lhes são inerentes, tendo também questões de credibilidade e de confiança próprias.

O relatório para a comunicação nas redes sociais publicado pelo Centers for Disease Control and Prevention (2011), refere-se às redes sociais e a outras tecnologias de comunicação como agregadoras de milhões de vozes para: (i) aumentar a disseminação da informação e o impacto da informação em saúde e segurança; (ii) aumentar o nível de audiências para facilitar a partilha de informação; (iii) expandir o alcance de forma a incluir públicos mais amplos e diversos; (iv) personalizar e reforçar as mensagens de saúde que mais facilmente podem ser adaptadas ou dirigidas a públicos específicos; (v) facilitar a comunicação interativa, conexão e participação do público; (vi) capacitar as pessoas para tomarem decisões mais seguras e saudáveis. Os resultados apresentados neste relatório têm implícito um fenómeno denominado "patient empowerment" um conceito que reflete uma mudança importante na relação paciente/médico: se anteriormente o paciente não questionava o médico nem a sua decisão, hoje estamos perante pacientes que entram nos consultórios com uma ideia formada sobre o seu estado de saúde e quais os tratamentos a aplicar (Shneiderman, 2003).

Os websites com informação de saúde, e em especial as redes sociais de saúde, permitem aos pacientes partilhar informação com base em experiências próprias ou de outros sobre temas como os sintomas, diagnósticos e tratamentos, efeitos adversos dos tratamentos, fontes de evidencia médica, experiências com prestadores de cuidados de saúde e opiniões sobre a sua qualidade (Griffiths et al., 2012). Evidencia-se assim a tomada de poder do paciente na relação paciente/prestadores de cuidados de saúde, que não só procura ativamente informação, como tem a possibilidade de discutir o assunto, partilhando ou questionando outros pacientes e tornando-se ele mesmo produtor de conteúdos de saúde. Este novo paradigma traz os seus desafios, incluindo questões de privacidade, de qualidade, de confiança e de credibilidade. Atualmente os fluxos são 
mais complexos e os papéis, no que respeita à comunicação e consumo de informação, são rotativos, o fluxo unidirecional das informações de saúde que partem dos profissionais de saúde como produtores e dos indivíduos como consumidores está a ser gradualmente substituído por um fluxo mais fluido e distribuído, onde todos, profissionais ou não, podem agir como consumidores e produtores de informação de saúde, conselhos e soluções (Burton, Tanner, \& Giraud-Carrier, 2012, p. 2). Com a facilidade de acesso a um enorme volume de informação sobre saúde disponível na Web, os consumidores poderão, eventualmente, passar a ignorar a intermediação de especialistas e a assumir assim a responsabilidade de avaliar a credibilidade da informação, sendo esta avaliação por parte do consumidor feita com base em variáveis como a autonomia e a motivação Eysenbach (2007), mas não colocando de parte as questões que pretendemos aprofundar neste estudo que trata o impacto da interface nessa mesma avaliação. $\mathrm{O}$ mesmo autor refere-se às caraterísticas do ambiente da mensagem, à apresentação ou atratividade da fonte assim como à sua credibilidade percebida como fatores primários decisores na credibilidade. Refere-se também aos novos meios de consumo de informação (que são também os novos intermediários) de "apomediários", que fazem um tipo de mediação que não implica necessariamente que se coloquem entre o consumidor ou entre os serviços e a informação. Especialmente para este estudo, importa a questão da desintermediação ou apomediação pois está diretamente relacionada com a perceção de credibilidade e de confiança online. Segundo o autor, a apomediação tem o potencial de aumentar a perceção de credibilidade principalmente em áreas como a saúde, em que os consumidores têm um sentimento de desconfiança do próprio sistema (Eysenbach, 2007).

\section{Proposta de dimensões da interface para o estudo da confiança e credibilidade online}

No decorrer desta investigação tornou-se necessária a sistematização da Interface em dimensões, para facilitar a análise da influência das mesmas na perceção de credibilidade e de confiança. Partindo de estudos anteriores, alguns deles referenciados ao longo deste artigo, e com o objetivo de analisar e caraterizar sistematicamente a Interface definimos cinco dimensões que acreditamos serem relevantes no processo de criação de credibilidade e confiança no desenho de uma Interface. Importa referir que estas dimensões não têm fronteiras estanques, elas partilham algumas propriedades e estão todas relacionadas entre si. 
Figura 1 Dimensões da Interface propostas pelas autoras

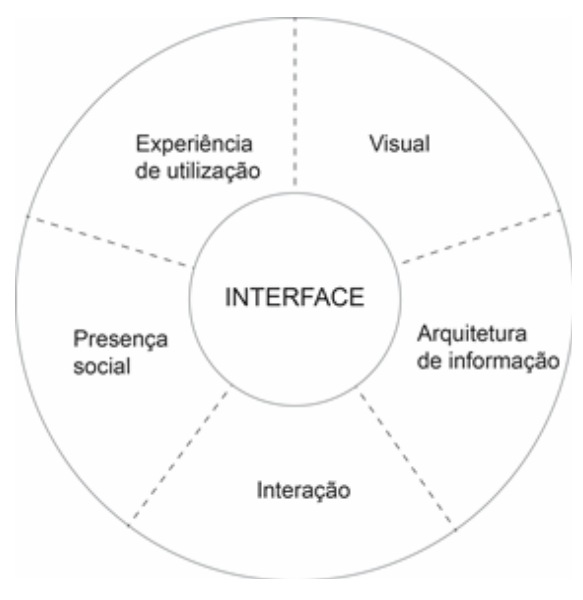

- Dimensão visual - garante a comunicação visual entre as partes envolvidas no processo de comunicação. É responsável pela materialização visual de todas as dimensões que compõem a Interface;

- Dimensão da arquitetura de informação - diz respeito à organização, estruturação e à designação dos conteúdos com o objetivo de ajudar os utilizadores a encontrarem e compreenderem os conteúdos;

- Dimensão da interação - responsável pela criação comportamentos ou funcionalidades de modo a proporcionar essencialmente interações entre as pessoas através dos computadores ou máquinas;

- Dimensão da presença social - permite a representação da presença, a conexão e a comunicação entre indivíduos e organizações;

- Dimensão da experiência da utilização - está relacionada com a perceção de utilização do uso ou da expetativa de uso de um produto ou serviço. Engloba aspetos como o afeto, a sensação e o significado dessa interação no dia a dia do utilizador.

Neste cenário desenvolveu-se uma proposta para a sistematização da problemática da perceção da confiança e da credibilidade através da Interface na e-Health, resultou na sua divisão em três conceitos principais: "a Interface", "a Credibilidade e a Confiança" e "o Utilizador”. Esta sistematização resulta da revisão da literatura e é apresentada sob a forma de um modelo de análise, desenhado a partir da proposta metodológica de Quivy e Campenhoudt (1998) na procura de constituir "a charneira entre a problemática fixada e 0 trabalho de elucidação sobre um campo de análise restrito e preciso" (pág. 15). Trata-se de uma abordagem que permite identificar, para cada conceito da problemática em estudo, um conjunto de dimensões e respetivos indicadores. 
Tabela 1 Modelo de análise proposto pelos autores

\begin{tabular}{|c|c|c|}
\hline & Dimensões & Indicadores \\
\hline \multirow[t]{15}{*}{ INTERFACE } & \multirow[t]{3}{*}{ Visual } & $\begin{array}{l}\text { Reconhecimento da presença da cor, da grelha e da tipografia } \\
\text { (Proeminência) }\end{array}$ \\
\hline & & $\begin{array}{l}\text { Reconhecimento da importância da cor, da grelha e da tipografia } \\
\text { na construção da sua credibilidade (Interpretação) }\end{array}$ \\
\hline & & Classificação da relevância de cada um dos elementos. \\
\hline & \multirow[t]{3}{*}{$\begin{array}{l}\text { Presença } \\
\text { social }\end{array}$} & $\begin{array}{l}\text { Reconhecimento da presença da componente social } \\
\text { (Proeminência) }\end{array}$ \\
\hline & & $\begin{array}{l}\text { Reconhecimento da importância da componente social na } \\
\text { construção da sua credibilidade (Interpretação) }\end{array}$ \\
\hline & & Classificação da relevância \\
\hline & \multirow{3}{*}{$\begin{array}{l}\text { Arquitetura } \\
\text { da } \\
\text { informação }\end{array}$} & Reconhecimento da presença da organização (Proeminência) \\
\hline & & $\begin{array}{l}\text { Reconhecimento da importância da organização da informação na } \\
\text { construção da sua credibilidade (Interpretação) }\end{array}$ \\
\hline & & Classificação da relevância \\
\hline & \multirow[t]{3}{*}{ Interação } & $\begin{array}{l}\text { Reconhecimento da presença da facilidade de navegação } \\
\text { (Proeminência) }\end{array}$ \\
\hline & & $\begin{array}{l}\text { Reconhecimento da importância da facilidade de navegação na } \\
\text { construção da sua credibilidade (Interpretação) }\end{array}$ \\
\hline & & Classificação da relevância \\
\hline & \multirow[t]{3}{*}{$\begin{array}{l}\text { Experiência } \\
\text { de utilização }\end{array}$} & $\begin{array}{l}\text { Reconhecimento da presença de uma boa experiência de utilização } \\
\text { (Proeminência) }\end{array}$ \\
\hline & & $\begin{array}{l}\text { Reconhecimento da importância da facilidade de navegação na } \\
\text { construção da sua credibilidade (Interpretação) }\end{array}$ \\
\hline & & Classificação da relevância \\
\hline \multirow{9}{*}{$\begin{array}{l}\text { CREDIBILIDADE E } \\
\text { CONFIANÇA }\end{array}$} & \multirow[t]{3}{*}{ Reputação } & Classificação da relevância \\
\hline & & Recomendado por conhecidos \\
\hline & & Presença de parceiros \\
\hline & \multirow[t]{3}{*}{ Familiaridade } & Classificação da relevância \\
\hline & & Recomendado por conhecidos \\
\hline & & Presença de parceiros \\
\hline & \multirow{3}{*}{$\begin{array}{l}\text { Natureza da } \\
\text { informação }\end{array}$} & Facilidade de compreensão \\
\hline & & Completude - ligação com outras fontes de informação - Perícia) \\
\hline & & Motivação \\
\hline \multirow[t]{4}{*}{ UTILIZADOR } & \multirow{4}{*}{$\begin{array}{l}\text { Acesso e } \\
\text { Participação }\end{array}$} & Frequência (Comunicação, Partilha e Consumo) \\
\hline & & Género \\
\hline & & Idade \\
\hline & & Condições de saúde \\
\hline
\end{tabular}




\section{Validação da proposta do modelo de análise para a perceção de confiança e credibilidade}

O plano que delineamos para aprofundar o conhecimento sobre a perceção de confiança e de credibilidade que tem como objetivo final propor um grupo de princípios de design para a credibilidade e confiança na e-Health, recorre à utilização de diferentes instrumentos (um inquérito por questionário, um Web browser plugin e sessões com eye tracker) que nos permitirão obter dados relativos à caraterização dos utilizadores e dos seus comportamentos, assim como, das suas avaliações relativamente à perceção que têm da influência da Interface na credibilidade e confiança dos websites de saúde que visitam. Estando o Web browser plugin ainda em desenvolvimento e as sessões com eye tracker ainda em fase de tratamento de dados iremos apresentar aqui resultados relativos ao inquérito por questionário.

Apresenta um conjunto de questões que permitem efetuar uma caraterização genérica dos respondentes, no que respeita à utilização da internet na partilha de informação de saúde e ao seu posicionamento sobre hábitos e perceções. Neste sentido, as perguntas foram formuladas na procura de articular as dimensões de acesso e participação, considerando diferentes indicadores (como o género, a idade, a motivação, as condições e saúde, a frequência de uso, partilha e comunicação de informação de saúde) com as dimensões da familiaridade, reputação e natureza da informação. O respondente é, portanto, inquirido igualmente acerca da facilidade de compreensão da informação pesquisada e da a sua completude, nomeadamente no que respeita à sua ligação a outras fontes de informação, explorando também o conceito de perícia. Sempre que necessário as questões referem-se a websites propostos por nós ou pelo respondente.

Numa segunda parte, e em função das respostas dadas na primeira parte, são colocadas ao respondente, em dois momentos distintos, questões específicas relativas à Interface de quatro websites

1 URL do website Portal do Utente: https://servicos. min-saude.pt/utente/

2 URL do website Manual Merk: http://www. manualmerck.net/

3 URL do website Biblioteca Virtual de Saúde: http:// www.bireme.br/php/ index.php

4 URL do website Medicina NET http://www. medicinanet.com.br/ selecionados com base no número de visitas na data de dezembro de 2013, sendo eles: Portal do Utente ${ }^{1}$, Manual Merk ${ }^{2}$, Biblioteca Virtual de Saúde ${ }^{3}$ e Medicina Net ${ }^{4}$. Importa referir que, nos casos em que o respondente indica um website, uma rede social ou um blog preferido para a consulta ou partilha de informação de saúde, esse website é utilizado nesta segunda parte do inquérito. Num primeiro momento explora-se a "credibilidade superficial" através da dimensão visual - nesta dimensão são questionados aspectos como a cor, a grelha e a tipografia. Num segundo momento, explora-se a "credibilidade adquirida”, nas dimensões de arquitetura de informação (relacionada com a organização visual da informação), a dimensão da presença 
social (relacionado com a presença de pistas sociais), a dimensão da interação (relacionado com a facilidade de navegação e da concretização dos objetivos do utilizador) e a dimensão da experiência de utilização (relacionada com a experiência de utilização integrando todas as componentes anteriores e questionando a satisfação do utilizador relativamente ao website). Este segundo momento, relativo à credibilidade adquirida, só se aplica nos casos em que o respondente indica familiaridade com o websites. Cada uma destas dimensões é analisada por três indicadores: a proeminência, a interpretação e a relevância. A proeminência e a relevância) serão analisados a partir das respostas do respondentes a questões abertas em que se pedem avaliações justificadas à perceção de credibilidade dos websites apresentados.

O inquérito por questionário foi desenvolvido e testado pelas autoras entre os meses de novembro de 2013 e fevereiro de 2014. Foi difundido online em Portugal através de redes sociais e via email para alunos das universidades do Porto e Aveiro (Portugal), durantes os meses de março, abril e maio de 2014. O desenvolvimento do inquérito e a recolha de dados foram feitos numa plataforma online da Universidade de Aveiro.

Obtivemos um total de 733 respostas das quais 219 completas. Após o tratamento de dados foram consideradas 154 respostas válidas; a diferença entre o total de respostas válidas e as completas refere-se aos participantes que responderam mas que não tinham práticas de pesquisa de informação de saúde na Web. A maioria dos respondentes é do sexo feminino e tem entre os 18 e os 25 anos de idade. Na sua maioria são estudantes universitários.

Os resultados aqui apresentados quanto à relevância das dimensões na perceção de credibilidade e de confiança apresentados no Quadro 1 resultam de uma análise estatística descritiva.

\section{Resultados e discussão}

Para a dimensão visual obtivemos 154 respostas no total, em relação às outras dimensões da Interface, como mencionado anteriormente, a segunda parte só estava disponível para os respondentes que indicaram familiaridade com um dos websites analisados anteriormente, ou caso tenham indicado um website preferido. Por esse motivo, existem diferentes números de respondentes para cada website. Em 154 respondentes, 99 indicaram familiaridade com os websites apresentados e 9 um website preferido perfazendo um total de 108 respostas. Os resultados apresentados confirmam a importância da dimensão visual para a perceção de confiança e credibilidade de um website apresentados em estudos anteriores (Fogg, Marshall, \& Laraki, 2001; Fogg, Marshall, \& Osipovich, 2000; Karimov et al., 2011; Robins et al., 2009). No entanto, a proposta que apresentamos para uma sistematização da interface em cinco dimensões permite-nos 
observar com mais detalhe quais as dimensões mais relevantes para a perceção de confiança e credibilidade. Como podemos verificar pelos resultados a dimensão da arquitetura da informação, a par com a experiência de utilização, são as dimensões mais relevantes para a perceção de confiança e credibilidade. O estudo conduzido por Karimov et al. (2011) já tinha avançado com resultados que apontavam para a relevância de outras dimensões que não só a visual para a perceção de confiança e credibilidade na interface; no entanto, estes resultados focam-se essencialmente na área do e-commerce e a divisão das dimensões não identifica a arquitetura de informação.

Quadro 1 Relevância das dimensões da Interface para a perceção de confiança e credibilidade

\begin{tabular}{|l|l|l|}
\hline Dimensão & $\begin{array}{l}\text { Relevância / Número de } \\
\text { respostas }\end{array}$ & Percentagem \\
\hline Visual & $102 / 154$ & $66,2 \%$ \\
\hline Arquitetura da informação & $96 / 108$ & $88,8 \%$ \\
\hline Interação & $91 / 108$ & $84,3 \%$ \\
\hline Presença social & $62 / 108$ & $57,4 \%$ \\
\hline Experiencia de utilização & $96 / 108$ & $88,8 \%$ \\
\hline
\end{tabular}

Os resultados aqui apresentados referem-se apenas a uma análise descritiva da influencia das dimensões na perceção de confiança e credibilidade. Estamos cientes de que será necessário cruzar estes resultados com as dimensões da reputação, familiaridade e natureza da informação apresentadas no modelo de análise. No entanto, acreditamos que os resultados apresentados até ao momento, e dada a caraterização dos nossos respondentes, permitem-nos inferir que a população jovem adulta entre os 18 e os 25 anos de idade com formação superior atribui grande importância à arquitetura de informação no que diz respeito à perceção de confiança e credibilidade em websites de saúde.

\section{Conclusões e considerações finais}

Com base na revisão da literatura desenvolvemos uma proposta de sistematização da Interface em cinco dimensões (visual, arquitetura da informação, interação, presença social e experiência de utilização) para a análise da confiança e da credibilidade em websites de informação de saúde. O trabalho que se desenvolveu para a sistematização da problemática resultou na sua divisão em três conceitos principais: "a Interface", "a Credibilidade e a Confiança" e "o Utilizador". Esta sistematização resulta da revisão da literatura e tem como referente um modelo de análise, desenhado a partir da proposta metodológica de Quivy e Campenhoudt (1998) a partir do qual foi possível associar um conjunto de dimensões e indicadores 
aos principais conceitos do estudo e que foram fundamentais para a estruturar quer o inquérito por questionário, quer o Web browser plugin (em fase de desenvolvimento) e a sessões com eye tracker.

No que diz respeito à classificação da relevância das dimensões da interface para a credibilidade e confiança os dados que obtivemos no inquérito por questionário indicam-nos que a dimensão visual tem uma forte influência na perceção de credibilidade, obtendo 102 respostas positivas em 154, e que entre os quatro elementos analisados (esquema de cores, tipografia, contraste e a organização de elementos na página) tipografia é o mais influente seguido pelo esquema de cores, contraste e a organização de elementos. No total, foram obtidas 108 respostas para as dimensões da arquitetura de informação, interação, presença social e de experiência de utilização. A influência das dimensões na credibilidade de uma perspectiva global, foi analisada a tentar compreender a relevância de cada uma das dimensões para a perceção de credibilidade a "arquitetura da informação" e a "experiência do utilizador" obtiveram 96 respostas positivas em 108 seguido pela "interação" com 91 e "presença social”, com 62 respostas positivas relativamente à sua influência na credibilidade e confiança. Como podemos verificar, a interface e as suas diferentes dimensões têm um papel preponderante na perceção da confiança e da credibilidade.

Acreditamos que com este estudo contribuímos para o aumento de conhecimento na área do design de informação com especial foco na área da saúde, não só através dos resultados que aqui apresentamos e dos que ainda pretendemos obter, como também com a proposta de sistematização da interface em cinco dimensões, o modelo de análise e os instrumentos aplicados, como o inquérito aplicado.

\section{Referências}

WBURTON, S. H., Tanner, K. W., \& Giraud-Carrier, C. G. (2012). Leveraging social networks for anytime-anyplace health information. Network Modeling Analysis in Health Informatics and Bioinformatics. doi:10.1007/s13721-012-0016-4

CENTERS FOR DISEASE CONTROL AND PREVENTION. (2011). The Health Communicator's Social Media Toolkit. Electronic Media, (July).

CHOW, W., \& Angie, N. (2006). A study of trust in e-shopping before and after first-hand experience is gained. Journal of Computer Information Systems.

DELONE, W. H., \& McLean, E. R. (1992). Information Systems Success: The Quest for the Dependent Variable, (4).

DU, Y., \& ZHAO, J. (2009). An empirical study of end-user trust in a web information system. 2009 International Conference on Information Managment, Innovation Management and Industrial Engineering, 561-564. doi:10.1109/ICIII.2009.293 
EYSENBACH, G. (2001). What is e-health? Journal of Medical Internet Research, 3(2), E20. doi:10.2196/jmir.3.2.e20

EYSENBACH, G. (2007). From Intermediation to Desintermediation and Apomediation: New Models for Consumers to Acess and Assess the Credibility of Health Information in the Age of Web 2.o. In Studies in Health Technology and Informatics, Volume 129: MEDINFO 2007 (pp. 162-166). IOS Press.

FOGG, B. (2003). Persuasive Technology - Using Computers to Change What We Think and Do. (A. Druin \& J. Hendler, Eds.). San Francisco: Morgan Kaufman.

FOGG, B., Marshall, J., \& Laraki, O. (2001). What makes Web sites credible?: a report on a large quantitative study. Conference on Human Factors in Computing Systems, 61-68.

FOGG, B., Marshall, J., \& Osipovich, A. (2000). Elements that affect web credibility: Early results from a self-report study. CHI'oo Extended ..., 287-288.

FOGG, B., Soohoo, C., Hall, C., Danielson, D. R., Tauber, E. R., Stanford, J., ... Marable, L. (2003). How Do Users Evaluate the Credibility of Web Sites? A Study with Over. ACM International Conference Proceeding Series, 1-15.

FORTNEY, J., Burgess, J., Bosworth, H., Booth, B., \& Kaboli, P. (2011). A reconceptualization of access for 21st century healthcare. Journal of General Internal Medicine, 26 Suppl 2, 639-47. doi:10.1007/s11606-011-1806-6

FURMAN, S. (2009, June 13). Building Trust. Usability.Gov. Department of Health and Human Services. Retrieved 5 July 2013, from http://www.usability.gov/ get-involved/blog/2009/o9/building-trust.html

GEFEN, D., \& Karahanna, E. (2003). Trust and TAM in Online Shopping: An Integarted Model. MIS Quarterly, 27(1), 51-90.

GRIFFITHS, F., Cave, J., Boardman, F., Ren, J., Pawlikowska, T., Ball, R., ... Cohen, A. (2012). Social networks - The future for health care delivery. Social Science \& Medicine (1982), 75(12), 2233-2241. doi:10.1016/j.socscimed.2012.08.023

KARIMOV, F. P., Brussel, V. U., Brengman, M., \& Hove, L. Van. (2011). The Effect of Website Design Dimensions on Initial Trust: A Synthesis of the empirical Literature. Journal of Electronic Commerce Research, 12(2), 272-301.

MORIN, E. (2011). Introdução ao Pensamento Complexo (4a Edição., Vol. 24 : 120).

QUIVY, R., \& Campenhoudt, L. Van. (1998). Manual de investigação em ciências sociais.

QUIVY, R., \& Campenhoudt, L. V. L. van. (2005). Manual de investigação em Ciências Sociais (4a Edição.). Lisboa: Gradiva - Publicações, Lda.

RASKIN, J. (2000). The Humane Interface: New Directions for Designing Interactive Systems. Addison-Wesley Professional.

RIEMENSCHNEIDER, C., Jones, K., \& Leonard, L. N. K. (2009). Web trust-A moderator of the web's perceived individual impact. Journal of Computer Information Systems.

ROBINS, D., Holmes, J., \& Stansbury, M. (2009). Consumer health information on the Web: The relationship of visual design and perceptions of credibility. Conference on Designing for User Experiences, 61(1), 13-29. doi:10.1002/asi 
SÁ, C. F. A. de. (2010). O que é um Interface? Da Entificação à Identificação do Interface equanto complexo Mediador. Universidade Nova de Lisboa.

SHNEIDERMAN, B. (2003). Leonardo's Laptop: Human Needs and the New Computing Technologies. Cambridge, MA, USA: MIT Press.

WANG, Y. D., \& Emurian, H. H. (2005). An overview of online trust: Concepts, elements, and implications. Computers in Human Behavior, 21(1), 105-125. doi:10.1016/j.chb.2003.11.008

\section{Sobre as autoras}

\section{Andreia Pinto de Sousa}

PHD Candidate in Communication and Information in Digital Platforms at University of Aveiro and Porto. She is also an Assistant Professor at the Department of Communication and Art, Polytechnic Institute of Viseu and at the Faculty of Communication, Arts and Information Technology, University Lusófona of Porto. She also works as a consultant in User Experience Designer for companies in the field of Design and Technology.

<souandreia@gmail.com>

\section{Ana Margarida Almeida}

PhD in Communication Sciences and Technologies and she has been developing research activities related to 'health promotion and health education through ICT', 'communication and health', 'digital inclusion', 'media for all' and 'agile projects development'. She is an Assistant Professor at the Department of Communication and Art, University of Aveiro, Portugal, she lectures in Communication Sciences and Technologies undergraduate, graduate and doctoral courses: 'New Communication Technologies bachelor degree', 'Multimedia Communications Master', 'Multimedia and Education PhD Program' and 'Information and Communication in Digital Platforms PhD Program". Under this scope, she has developed several supervising activities of MSc and PhD students, highly related to her main research interests and she has been involved in different research projects and scientific initiatives. She has several papers and book chapters published and she is also a reviewer in several national and international journals. <marga@ua.pt>

Artigo recebido em 22 mar. 2015, aprovado em 18 jun. 2015. 